\title{
Evaluación de la adaptación de los ítems de consumo del AUDIT para mejorar el cribado de Binge Drinking en universitarios
}

\section{Evaluation of AUDIT Consumption Items New Adaptation to Improve the Screening of College Students Binge Drinking}

\author{
Patricia Motos-Sellés*, María-Teresa Cortés-Tomás**, José-Antonio Giménez-Costa**. \\ * Área de Educación. Universidad Internacional de Valencia, Valencia, España. ** Departamento de Psicología Básica, Facultad \\ de Psicología. Universidad de Valencia, Valencia, España.
}

\section{Resumen}

La importante presencia del Binge Drinking (BD) entre estudiantes universitarios, junto con las consecuencias asociadas al mismo y los cambios experimentados en los últimos años en su conceptualización, hacen necesario revisar la utilidad de los instrumentos de cribado para detectar este patrón de consumo. Este estudio examina la utilidad de una adaptación del AUDIT propuesta por Cortés, Giménez, Motos y Sancerni (2017a).

Una muestra de estudiantes universitarios cumplimentó el AUDIT, los ítems 2 y 3 revisados (A2r y A3r), y un autoinforme semanal de su consumo de alcohol. A partir de la cantidad máxima de alcohol consumido en una ocasión y de la frecuencia de dicho consumo en los últimos seis meses se clasificaron los jóvenes como BD o no-BD. Se examinaron las puntuaciones del AUDIT, AUDIT-C y de los ítems A2r+A3r (AR2I).

Los resultados obtenidos con 605 universitarios (18-21 años/55,2\% mujeres) indican que 449 cumplen criterios de BD. Los ítems A2r y A3r, adaptados a una definición más consensuada de $\mathrm{BD}$, identifican el $98 \%$ de los estudiantes BD cuando se usa un punto de corte $\geq 3$ en mujeres $\mathrm{y} \geq 4$ en varones, con valores óptimos de sensibilidad y especificidad.

Esta adaptación realizada, que incluye menor número de ítems, identifica a los universitarios BD de manera más precisa. Se confirma la necesidad de ajustar ambos ítems de consumo de acuerdo al patrón de ingesta $\mathrm{BD}$ que realizan los estudiantes universitarios mejorando notablemente su detección y facilitando un abordaje temprano. Palabras clave: Binge drinking; Universitarios; AUDIT; Cribado de alcohol; ROC.

\section{Abstract}

The strong presence of Binge Drinking (BD) amongst university students, as well as the consequences associated with the same and the changes taking place over recent years regarding its conceptualization make it necessary to examine the usefulness of screening instruments used to detect this drinking pattern. This study examines the usefulness of a briefer adaptation of the AUDIT proposed by Cortés, Giménez, Motos, and Sancerni (2017a).

College students self-administered the AUDIT, the revised items 2 and 3 (A2r and A3r), and completed a weekly self-report of their alcohol intake. BD was classified according to the amount consumed and the frequency of that consumption over the past six months. The AUDIT, AUDIT-C and items A2r+A3r (AR2I) were examined.

The results obtained from a sample of 605 college students (1821 years old $/ 55.2 \%$ female) indicate that 449 meet the BD criteria. Items A2r and A3r, adapted to the most consensual definition of BD, were found to identify $98 \%$ of BD college students when using a cutoff point of $\geq 3$ in females and $\geq 4$ in males with optimum levels of sensitivity and specificity.

The new adaptation, which includes fewer items, identifies BD college students more accurately. This confirms the need to adjust both consumption items from the model according to the pattern of consumption in college students to detect $\mathrm{BD}$ more precisely and as soon as possible.

Keywords: Binge Drinking; Undergraduate; AUDIT; Alcohol Screening; ROC. 
E 1 patrón de consumo de alcohol más generalizado entre los jóvenes europeos es el denominado Binge Drinking (BD), término adaptado al castellano como Episodio de Consumo Intensivo de Alcohol (Ministerio de Sanidad y Consumo [MSC], 2008). En Europa, dos de cada diez jóvenes con edades entre los 14 y 24 años reconocen realizar este tipo de consumo (Unión Europea, 2010). En el caso de los jóvenes españoles (Observatorio Español de las Drogas y las Adicciones [OEDA], 2017), la mayor incidencia se sitúa en el intervalo de edad entre 20 y 29 años, estando presente en aproximadamente el $35 \%$ de hombres y $23 \%$ de mujeres. Si bien, al revisar la prevalencia de $\mathrm{BD}$ entre los menores de edad durante el último año (OEDA, 2018), esta oscila desde un 14\% en menores de 14 años hasta un $56 \%$ en jóvenes de 18 años. Desafortunadamente, tanto en España como en Europa en general, los cambios en este patrón de consumo entre los menores de edad han sido menos pronunciados y se han observado únicamente entre varones (42\% a $37 \%$ ), con las tasas generales bajando un punto porcentual (36\% a $35 \%)$ en los últimos 20 años (European School Survey Project on Alcohol and Other Drugs [ESPAD], 2016). Además, se advierte desde hace tiempo (Kuntsche et al., 2011; Simons-Morton et al., 2009), y ahora se confirma, un cierre de la brecha de género del consumo excesivo de alcohol entre los adolescentes. En general, las tasas de BD aumentaron entre chicas jóvenes, lo que resulta en una reducción de las diferencias de género a lo largo del tiempo (ESPAD, 2016; OEDA, 2018).

No es posible separar este patrón de consumo de alcohol de las consecuencias que genera entre los jóvenes. En general, destacan alteraciones y problemas en diferentes grados y en diferentes ámbitos, desde el ámbito académico o profesional, al de relaciones interpersonales, la exposición a conductas sexuales de riesgo, conducir bajo la influencia del alcohol, participar en peleas, sufrir lesiones, incurrir en problemas legales, progresar con mayor facilidad en el proceso adictivo o incluso causar daños a terceros (Barnet et al., 2014; Brewer y Swahn, 2005; Cortés, Motos y Giménez, 2015; Hingson, Heeren, Zakocs, Winter y Wechsler, 2003; Mallett, Varvil-Weld, Turrisi y Read, 2011; McKetin, Chalmers, Sunderland y Bright, 2014; Shield, Gmel, Patra y Rehm, 2012).

La elevada presencia de este patrón de consumo entre los jóvenes, unido a los cambios que se están experimentando en el mismo en cuanto a la equiparación por sexos y las consecuencias que genera, justifican la necesidad de disponer de instrumentos de cribado que faciliten su detección en diferentes ámbitos (Atención Primaria, departamentos de traumatología, servicios de salud universitarios, etc.) y la posterior derivación del joven al recurso asistencial más adecuado (Clark y Moss, 2010).

El AUDIT es un instrumento de cribado utilizado para identificar el Binge Drinking (BD) en estudiantes universita- rios (Hagman, 2016; Seguel, Santander y Alexandre, 2013). Se recomienda el uso de sus versiones abreviadas, dada su mayor efectividad (Barry, Chaney, Stellefson y Dodd, 2015; Clark, Gordon, Ettaro, Owens y Moss, 2010; de Meneses-Gaya, Zuardi, Loureiro y Crippa, 2009). De estas versiones abreviadas, cabe destacar el AUDIT-C, al contar con mejores propiedades psicométricas para la población universitaria que la escala completa (Barry et al., 2015; Cortés, Giménez, Motos y Sancerni, 2016; DeMartini y Carey, 2012; Kelly, Donovan, Chung, Bukstein y Cornelius, 2009).

Pero el AUDIT y sus versiones reducidas utilizan una operacionalización poco precisa del $\mathrm{BD}$, lo que dificulta poder identificarlo con precisión. De los tres ítems relacionados con la ingesta de alcohol, solo el tercero trata de reflejar un consumo en atracón (¿ל Con qué frecuencia consume 6 o más bebidas por día?) pero está muy alejado de lo que se considera un Consumo Intensivo de Alcohol en la actualidad (Cortés y Motos, 2015; Mota et al., 2010).

Otro problema añadido es que muchos de los estudios que han aplicado el AUDIT o sus versiones abreviadas han medido BD de diversas maneras, recurriendo en la mayoría de los casos al número de consumiciones ingeridas sin tener en cuenta la graduación de la bebida, o no detallando el número de horas de duración de la ingesta, o evaluando solo el consumo de la última semana, o utilizando criterios del DSM para Trastorno por Consumo de Sustancias (Chung, Colby, Barnett y Monti, 2002; Díaz Martínez et al., 2009; Thomas y McCambridge, 2008). Además, escasos estudios han tenido en cuenta la alta heterogeneidad de este grupo de consumidores.

Esta diversidad justifica la necesidad de ser muy cautos a la hora de comparar resultados entre las diferentes investigaciones, al tiempo que exige la necesidad de un mayor rigor en la operacionalización del BD y de los instrumentos que tratan de dar cuenta del mismo.

La necesidad de mejorar los instrumentos de medida ha propiciado que se hayan propuesto adaptaciones del AUDIT. Inicialmente, se evaluaron nuevas combinaciones de ítems diferentes a las versiones abreviadas tradicionales, tratando de identificar aquellas que detectaban de manera más precisa los consumidores BD (Babor, Higgins-Biddle y Robaina, 2017). En el caso de McCambridge y Thomas (2009), informaron que la mejor combinación sería la compuesta por los ítems 3, 5 y 8, mientras que Bowring, Gouillou, Hellard y Dietze (2013) concluyeron que la mejor agrupación era la de los ítems 3, 4, 8 y 9 .

No obstante, han resultado más efectivos los intentos de reajustar la redacción de los ítems o sus escalas de respuesta a una definición más precisa del BD. Destacar en esta línea diferentes investigaciones que comparten la eliminación del primer ítem del AUDIT-C debido a su baja correlación con la escala total (Gmel, Hebb y Rehm, 2001; McCambridge y Thomas, 2009). 
Blank, Connor, Gray y Tustin (2015) propusieron utilizar sólo los ítems 2 y 3 (los que mejor explican BD en universitarios) a la vez que aumentar el número de opciones en las respuestas. Otros estudios recomendaron modificar la redacción de estos dos ítems referidos al consumo. Para el ítem 2, García, Novalbos, Martínez y O’Ferrall (2016) sugirieron limitar el intervalo temporal de consumo a "una única ocasión de consumo" (en lugar de "un día cualquiera”). En cuanto al ítem 3, en algunos casos, se sugirió una reducción del número de bebidas (cinco o más bebidas en una única ocasión de consumo -Kokotailo et al., 2004-; cuatro o más bebidas para mujeres y cinco o más bebidas para hombres -Olthuis, Zamboanga, Martens y Ham, 2011) y en otros se sugirió transformar el número de bebidas a Unidades de Bebida Estándar (UBE), según el país de origen (García et al., 2016). La introducción de este tipo de modificaciones produjo aumentos en los niveles de sensibilidad (entre 0,82 y 0,87 ) y de especificidad (entre 0,87 y 0,92 ), en comparación con la escala tradicional (Blank et al., 2015; McCambridge y Thomas, 2009).

No obstante, ninguna de estas sugerencias ha gozado de aceptación generalizada por no contemplar una definición ajustada del BD.

Recientemente, Cortés, Giménez, Motos y Sancerni (2017a) adaptaron la redacción de los ítems 2 y 3 a la definición con mayor consenso de $\mathrm{BD}$, que atiende a criterios más precisos de operalización de esta conducta, según la investigación (Cortés y Motos, 2015; Courtney y Polich, 2009; Parada et al., 2011), incluyendo aspectos de género, intervalo temporal del consumo, y equivalencias a la UBE Española. De este modo, se define BD como el consumo de siete o más UBE para hombres, o seis o más para mujeres, en un periodo de dos horas, al menos una vez en los últimos seis meses (Cortés et al., 2016; Cortés, Giménez, Motos, Sancerni y Cadaveira, 2017b).

Atendiendo a estos criterios, el ítem 3 se enunció como: En los últimos seis meses, ¿̇cuántos días al mes, como media, realizaste BD (siete o más UBE para hombres o seis o más UBE para mujeres, durante dos horas)? La escala de respuesta también se adaptó en función de los resultados obtenidos en investigaciones previas realizadas con menores y estudiantes universitarios (Cortés et al., 2017a; Hagman, 2016; Patrick et al., 2013) (Tabla 1).

También se mejoró la redacción de ítem 2, cambiando el número de consumiciones por el número de UBE españolas ingeridas en un día de consumo: ¿Cuántas UBE tiendes a consumir en un día cuando bebes alcohol? (Tabla 1).

$\mathrm{Al}$ probar esta adaptación (AR2I) con menores (14-17 años) consumidores de alcohol, se halló un área bajo la curva ROC (Característica Operativa del Receptor) de 0,898, identificando al $94 \%$ de los consumidores BD con un punto de corte de 5 .

El objetivo de este estudio se centra en examinar las propiedades psicométricas (sensibilidad y especificidad) del AUDIT, el AUDIT-C y de la adaptación AR2I, tratando de identificar aquel que mejor clasifique a los $\mathrm{BD}$ en población universitaria (18-21 años), considerando la variable de género.

A partir de los resultados obtenidos en el estudio previo de Cortés et al. (2017a) sobre la efectividad de la adaptación del AR2I en la identificación de adolescentes BD, se plantea la hipótesis de que será este instrumento el que identifique a un mayor número de universitarios BD.

\section{Método}

\section{Participantes}

Se realizó un muestreo estratificado de los estudiantes de la Universitat de València. Se seleccionó la licenciatura o diplomatura de cada área de conocimiento (Ciencias Básicas, Sociales, de la Salud, de la Educación y Humanidades) con mayor número de matriculados, respondiendo el cuestionario el grupo más numeroso de cada curso. Participaron 605 estudiantes, todos con nacionalidad española (334 mujeres/55,2\%). Las edades estaban comprendidas entre los 18 y 21 años, con una edad media de 19,33 años $(\mathrm{SD}=1,15)$. En ningún caso cumplían criterios diagnósticos para recibir tratamiento por conducta adictiva. De los encuestados, el 74,21\% (449) cumplió los criterios de BD, en proporción similar entre hombres $(44,5 \%, 200)$ y mujeres $(55,5 \%, 249)\left(\mathrm{X}^{2}=, 044 ; p=, 834\right)$.

Los cuestionarios se cumplimentaron durante el curso académico 2014 en las aulas docentes y durante su horario lectivo (mañana o tarde), contando en todos los casos con la presencia de algún miembro del equipo de investigación que confirmaba que todos los ítems habían sido respondidos. La participación fue anónima y voluntaria. El estudio se realizó cumpliendo con la legislación española (aprobado por el Ministerio de Educación) y el Código de Ética para estudios humanos del Comité de Ética de la Universidad de Valencia. Los estudiantes firmaron una hoja de consentimiento informado.

\section{Variables e instrumentos}

Los parámetros relacionados con el consumo de alcohol (edad de inicio de consumo, número y tipo de bebidas y

Tabla 1. Nueva redacción de los ítems 2 y 3.

A2r ¿Cuántas UBE tiendes a consumir en un día cuando bebes alcohol?

(0) 1 о 2; (1) 3 о 4; (2) 5 о 6; (3) 7 a 9; y (4) 10.

A3r En los últimos seis meses, ¿cuántos días al mes, como media, realizaste BD (siete o más UBE para hombres o seis o más UBE para mujeres, durante dos horas)?

(0) Nunca; (1) De manera esporádica -menos de una vez al mes-; (2) entre 1 y 4 veces; (3) entre 5 y 8 veces; (4) entre 9 y 12 veces; (5) 13 o más veces. 
periodos temporales durante los cuales se consumía alcohol) se evaluaron mediante un diario autoinformado. Los consumos fueron convertidos a UBE según la definición de UBE española -1 bebida destilada $=2$ UBE; 1 bebida fermentada = 1 UBE- (Rodríguez-Martos, Gual y Llopis, 1999). La recodificación de todos los consumos registrados permitió identificar la ocasión de mayor consumo realizada por cada estudiante (mayor número de UBE de alcohol consumidas en una ocasión de BD). Teniendo en cuenta esta variable, los estudiantes se clasificaron como no-BD, o BD (aquellos que consumieron $\geq 7$ UBE de España para hombres o $\geq 6$ UBE de España para mujeres).

La frecuencia de $\mathrm{BD}$ se obtuvo preguntando el número de días que realizó $\mathrm{BD}$ en los últimos seis meses.

Los ítems A2r+A3r se operacionalizaron según la descripción que figura en la Tabla 1.

También cumplimentaron el AUDIT (versión en español validada por Contel Guillamón, Gual Solé y Colom Farran, 1999) obteniéndose la puntuación del AUDIT total (la suma de los 10 ítems originales) y la del AUDIT-C (la suma de los primeros tres ítems originales). También se cumplimentó el AR2I, obteniéndose la puntuación A2r + A3r.

En este estudio, la consistencia interna de AR2I fue más alta $(0,90)$ que la del AUDIT-C $(0,80)$ y la del AUDIT $(0,71)$.

\section{Análisis de datos}

Siguiendo la metodología propuesta por Cortés et al. (2016, 2017a, 2017b), se llevaron a cabo dos análisis de conglomerados (uno por sexo) con los estudiantes universitarios $\mathrm{BD}$, en base al valor del número de UBE consumidas en una ocasión de BD y la frecuencia de consumo en los últimos seis meses. En ambos casos, el procedimiento de extracción fue en dos fases, permitiendo una clasificación natural de los sujetos en grupos diferentes.

Se llevó a cabo también un análisis MANOVA con sus correspondientes pruebas a posteriori, utilizando los cuatro grupos obtenidos en los conglomerados y los grupos no$\mathrm{BD}$ como variables independientes (VI) para determinar si existían diferencias en el número de UBE consumidas y la frecuencia de consumo en los últimos seis meses utilizadas como variables dependientes (VD).

El área bajo la curva ROC se calculó según el método propuesto por Hanley y McNeil (1983), que ofrece una representación gráfica del rendimiento del clasificador.

Para determinar el punto de corte óptimo del AUDIT, se primó el minimizar los falsos negativos y mejorar la detección de jóvenes que realizan en BD. Por ello se priorizaron los puntos de corte que maximizaban la sensibilidad.

Todos los análisis se realizaron con el paquete estadístico IBM SPSS-22.

\section{Resultados}

El análisis de conglomerados entre mujeres BD ofreció dos grupos: el BD1M ( $\mathrm{n}=169$ ), que consumió 8,44 UBE en una ocasión y que realizó BD durante 22,85 días en los últimos seis meses, y el BD2M $(\mathrm{n}=80)$, que consumió

Tabla 2. Prueba de Games-Howell a posteriori.

\begin{tabular}{|c|c|c|c|c|c|c|}
\hline (I) Agrupaciones_solo_BD & (J) Agrupaciones_solo_BD & $\begin{array}{r}\text { Diferencia } \\
\text { de medias }(I-J)\end{array}$ & SE & Sig. & \multicolumn{2}{|c|}{$\begin{array}{r}\text { Intervalo de confianza 95\% } \\
\text { (Extremo inferior - Extremo superior) }\end{array}$} \\
\hline \multicolumn{7}{|l|}{ UBE } \\
\hline \multirow[t]{5}{*}{ BD1M } & $\mathrm{BD} 2 \mathrm{M}$ & $-4,968\left(^{\star}\right)$ & ,469 &, 001 & $-6,27$ & $-3,67$ \\
\hline & BD1H & $-3,606\left(^{\star}\right)$ & ,298 & ,001 & $-4,42$ & $-2,79$ \\
\hline & $\mathrm{BD} 2 \mathrm{H}$ & $-16,687\left(^{\star}\right)$ & ,738 &, 001 & $-18,79$ & $-14,59$ \\
\hline & NOBDM & $4,867\left(^{\star}\right)$ & ,211 &, 001 & 4,26 & 5,47 \\
\hline & $\mathrm{NOBDH}$ & $4,079\left(^{\star}\right)$ & ,237 &, 001 & 3,40 & 4,76 \\
\hline \multirow[t]{4}{*}{ BD2M } & $\mathrm{BD} 1 \mathrm{H}$ & 1,363 &, 509 &, 063 &,- 04 & 2,77 \\
\hline & $\mathrm{BD} 2 \mathrm{H}$ & $-11,719\left(^{\star}\right)$ & ,846 & ,001 & $-14,09$ & $-9,35$ \\
\hline & NOBDM & $9,836\left(^{\star}\right)$ & ,463 & ,001 & 8,49 & 11,18 \\
\hline & $\mathrm{NOBDH}$ & $9,047\left(^{\star}\right)$ & ,476 & 001 & 7,67 & 10,43 \\
\hline \multirow[t]{3}{*}{ BD1H } & $\mathrm{BD} 2 \mathrm{H}$ & $-13,081(*)$ & ,764 & ,001 & $-15,24$ & $-10,92$ \\
\hline & NOBDM & $8,473\left(^{\star}\right)$ & ,288 & ,001 & 7,64 & 9,30 \\
\hline & $\mathrm{NOBDH}$ & $7,685\left(^{*}\right)$ & ,308 & ,001 & 6,80 & 8,57 \\
\hline \multirow[t]{2}{*}{$\mathrm{BD} 2 \mathrm{H}$} & NOBDM & $21,554\left(^{*}\right)$ & ,734 & ,001 & 19,36 & 23,75 \\
\hline & $\mathrm{NOBDH}$ & $20,766\left(^{\star}\right)$ & ,742 & ,001 & 18,55 & 22,98 \\
\hline NOBDM & $\mathrm{NOBDH}$ &,$- 789\left(^{\star}\right)$ & ,224 & ,008 & $-1,44$ &,- 14 \\
\hline
\end{tabular}


Consumo total días en 6 meses

\begin{tabular}{|c|c|c|c|c|c|c|}
\hline \multirow[t]{5}{*}{ BD1M } & $\mathrm{BD} 2 \mathrm{M}$ & $-22,873\left({ }^{\star}\right)$ & 1,661 & ,001 & $-27,47$ & $-18,28$ \\
\hline & BD1H & $-11,385\left(^{\star}\right)$ & 1,464 & ,001 & $-15,41$ & $-7,36$ \\
\hline & $\mathrm{BD} 2 \mathrm{H}$ & $-24,684\left(^{\star}\right)$ & 2,264 & ,001 & $-31,06$ & $-18,24$ \\
\hline & NOBDM & $6,334\left(^{\star}\right)$ & 1,534 &, 001 & 1,90 & 10,77 \\
\hline & $\mathrm{NOBDH}$ & $5,979\left(^{\star}\right)$ & 1,776 &, 013 & ,82 & 11,14 \\
\hline \multirow[t]{4}{*}{$\mathrm{BD} 2 \mathrm{M}$} & BD1H & $11,488\left(^{\star}\right)$ & 1,923 & ,001 & 6,19 & 16,78 \\
\hline & $\mathrm{BD} 2 \mathrm{H}$ & $-1,775$ & 2,584 & ,959 & $-9,00$ & 5,45 \\
\hline & NOBDM & $29,207\left(^{\star}\right)$ & 1,977 & ,001 & 23,51 & 34,91 \\
\hline & $\mathrm{NOBDH}$ & $28,852\left(^{*}\right)$ & 2,170 & ,001 & 22,59 & 35,12 \\
\hline \multirow[t]{3}{*}{ BD1H } & $\mathrm{BD} 2 \mathrm{H}$ & $-13,263\left({ }^{\star}\right)$ & 2,463 & ,001 & $-20,16$ & $-6,36$ \\
\hline & NOBDM & $17,720\left(^{\star}\right)$ & 1,815 & ,001 & 12,50 & 22,94 \\
\hline & $\mathrm{NOBDH}$ & $17,364\left(^{\star}\right)$ & 2,023 & ,001 & 11,53 & 23,20 \\
\hline \multirow[t]{2}{*}{$\mathrm{BD} 2 \mathrm{H}$} & NOBDM & $30,982\left(^{\star}\right)$ & 2,505 & ,001 & 23,64 & 38,32 \\
\hline & $\mathrm{NOBDH}$ & $30,627\left(^{\star}\right)$ & 2,660 &, 001 & 22,86 & 38,39 \\
\hline NOBDM & $\mathrm{NOBDH}$ &,- 356 & 2,075 & 1,000 & $-6,35$ & 5,64 \\
\hline \multicolumn{7}{|c|}{ Años de consumo } \\
\hline \multirow[t]{5}{*}{ BD1M } & $\mathrm{BD} 2 \mathrm{M}$ &,$- 69667\left(^{\star}\right)$ & ,21749 & ,020 & $-1,3249$ &,- 0684 \\
\hline & $\mathrm{BD} 1 \mathrm{H}$ & ,21583 & ,19221 & ,872 &,- 3355 & ,7671 \\
\hline & $\mathrm{BD} 2 \mathrm{H}$ &,- 28417 & ,32799 & ,953 & $-1,2556$ & ,6872 \\
\hline & NOBDM & ,28200 & ,23387 & ,833 &,- 3938 & ,9578 \\
\hline & $\mathrm{NOBDH}$ &, $93379\left(^{\star}\right)$ & ,25042 &, 004 & ,2074 & 1,6602 \\
\hline \multirow[t]{4}{*}{$\mathrm{BD} 2 \mathrm{M}$} & BD1H &, $91250\left(^{\star}\right)$ & ,23983 &, 003 & ,2217 & 1,6033 \\
\hline & $\mathrm{BD} 2 \mathrm{H}$ & ,41250 & ,35798 & ,857 &,- 6374 & 1,4624 \\
\hline & NOBDM &, $97868\left(^{\star}\right)$ &, 27435 & ,006 & ,1874 & 1,7699 \\
\hline & $\mathrm{NOBDH}$ & $1,63046\left(^{\star}\right)$ & ,28859 & ,001 & ,7966 & 2,4643 \\
\hline \multirow[t]{3}{*}{ BD1H } & $\mathrm{BD} 2 \mathrm{H}$ &,- 50000 & ,34321 & ,692 & $-1,5103$ &, 5103 \\
\hline & NOBDM &, 06618 &, 25478 & 1,000 &,- 6679 & ,8003 \\
\hline & $\mathrm{NOBDH}$ & ,71796 & ,27005 &, 090 &,- 0625 & 1,4984 \\
\hline \multirow[t]{2}{*}{$\mathrm{BD} 2 \mathrm{H}$} & NOBDM &, 56618 & ,36817 & ,641 &,- 5109 & 1,6433 \\
\hline & $\mathrm{NOBDH}$ & $1,21796\left(^{\star}\right)$ & ,37889 & ,023 & ,1111 & 2,3248 \\
\hline NOBDM & $\mathrm{NOBDH}$ & ,65178 & ,30113 & ,261 &,- 261 & 1,5212 \\
\hline
\end{tabular}

* Nota. Las diferencias de medias tienen nivel de significancia a o,05.

$\mathrm{BD}=\mathrm{Binge}$ Drinking; $\mathrm{SE}=$ Error estándar; $\mathrm{BD} 1 \mathrm{M}=\mathrm{Grupo}$ uno de $\mathrm{BD}$, mujeres; $\mathrm{BD} 2 \mathrm{M}=\mathrm{Grupo}$ dos de $\mathrm{BD}$, mujeres; $\mathrm{BD} 1 \mathrm{H}=\mathrm{Grupo}$ uno de $\mathrm{BD}$, hombres; $\mathrm{BD} 2 \mathrm{H}=\mathrm{Grupo}$ dos de $\mathrm{BD}$, hombres; NOBDM=Grupo de no BD, mujeres; $\mathrm{NOBDH}=$ Grupo de no BD, hombres.

13,41 UBE por ocasión con una media de 45,73 episodios de $\mathrm{BD}$ en los últimos seis meses. En el caso de hombres $\mathrm{BD}$, se obtuvieron dos grupos: el BD1H $(\mathrm{n}=160)$, que consumió 12,04 UBE de media en cada ocasión y realizó BD durante 34,24 días durante los últimos seis meses, y el BD2H ( $\mathrm{n}=40$ ), que consumió 25,13 UBE en cada ocasión, con una media de 47,5 episodios de BD en los últimos seis meses.

Los participantes que no cumplieron los criterios para ser considerados BD fueron clasificados en dos grupos en función del sexo. El grupo de mujeres -NOBDF- $(\mathrm{n}=85)$ que consumió 3,57 UBE en una ocasión, con una frecuen- cia de 16,52 días en los últimos seis meses, y el grupo de hombres -NOBDH- $(\mathrm{n}=71)$, que consumió 4,36 UBE por ocasión unos 16,87 días durante el mismo periodo.

El análisis MANOVA realizado entre los cuatro grupos BD y los dos grupos NOBD (Tabla 2) mostró diferencias significativas en el número de UBE consumidas y en la frecuencia de consumo en los últimos seis meses.

Según la Tabla 2, los cuatro grupos BD consumieron cantidades significativamente más altas y con mayor frecuencia que los grupos NOBD. No se encontraron diferencias significativas entre los grupos NOBD en ninguna de las dos variables. 


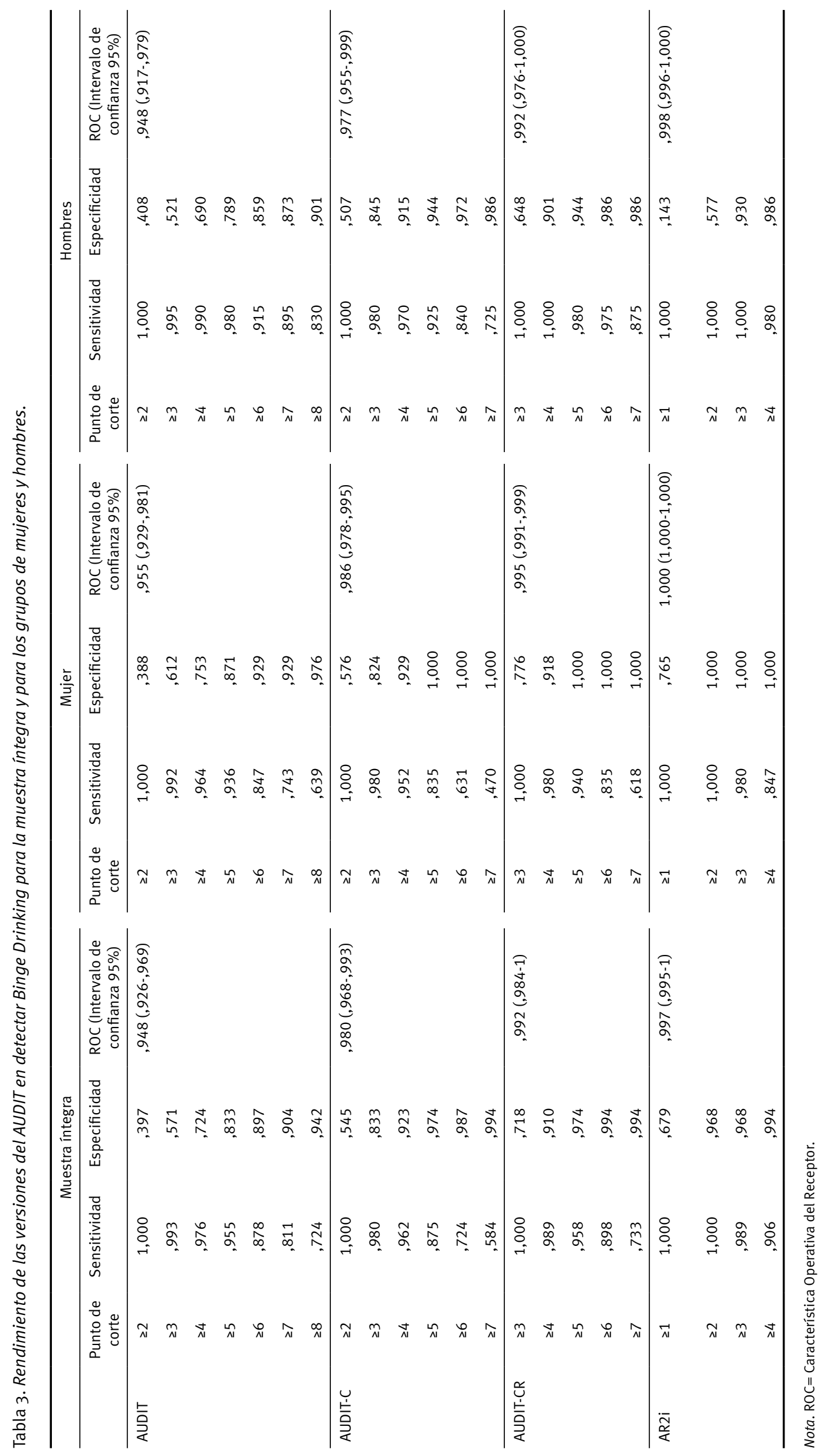


$\mathrm{Al}$ comparar los cuatro grupos $\mathrm{BD}$, se aprecia que los subgrupos que consumieron las cantidades más altas de UBE (BD2M y BD2H) también consumieron con mayor frecuencia que los otros dos subgrupos (BD1M y BD1H). Al considerar el sexo de ambos subgrupos, fueron los hombres los que consumieron mayores cantidades, en comparación con sus respectivos subgrupos de mujeres. No se hallaron diferencias en cuanto a la variable de frecuencia de consumo, con la excepción de ambos subgrupos de BD de menor intensidad, en los cuales los hombres realizaron BD con más frecuencia que las mujeres en los últimos seis meses.

Como muestra la Tabla 3, las puntuaciones del AUDIT y el AUDIT-C presentan valores óptimos en el área bajo la curva ROC; no obstante, son más bajos que los valores obtenidos en las versiones adaptadas.

La redefinición de los ítems 2 y 3 (AR2i) ofrece un área bajo la curva ROC mayor tanto para la muestra completa de BD como para cada uno de los sexos. Con un punto de corte de $\geq 3$ se detectan el 98,9\% de todos los BD (sensibilidad) y el $96,8 \%$ de los no-BD (especificidad). Con los puntos de corte de $\geq 3$ para mujeres y de $\geq 4$ para hombres, se quedan sin detectar solo el $2 \%$ de los BD de cada grupo.

\section{Discusión}

Estudios recientes (Cortés et al., 2016, 2017a, 2017b; Patrick et al., 2013; Read, Beattie, Chamberlain y Merrill, 2008) aluden a la heterogeneidad existente entre los jóvenes $\mathrm{BD}$, ya que la propia definición de $\mathrm{BD}$ solo indica un mínimo a partir del cual identificar un BD, sin atender a diferentes niveles de gravedad. En la misma línea, este estudio identifica grupos de consumidores BD que, considerando tanto las cantidades consumidas (duplican el mínimo de la definición de BD) como la frecuencia de participación en los episodios (dos veces por semana), incrementan el riesgo de experimentar consecuencias negativas como resultado de dicho consumo de alcohol. Este hecho se agrava notablemente cuando el consumo se inicia a una edad muy temprana, como en el caso de la muestra de este estudio que se iniciaron en el consumo de alcohol entre los 13 y los 15 años. Según los datos de la última encuesta nacional (OEDA, 2018), la edad media de inicio se sitúa alrededor de los 14 años, considerada un momento crítico por incrementar de manera notoria la posible aparición de consecuencias negativas en periodos posteriores (Hingson, Zha y Weitzman, 2009; Jenkins et al., 2011; Pilatti, Caneto, Garimaldi, Del Valle y Pautassi, 2013). De ahí la mayor necesidad de plantear intervenciones ajustadas para paliar o prevenir estas consecuencias (Vargas-Martínez, Trapero-Bertran, Gil-García y Lima-Serrano, 2018).

Entre los grupos con mayor intensidad de BD, se observa un consumo mucho más acentuado en hombres que en mujeres, ya que ellos llegan a triplicar la cantidad de alcohol mientras que ellas lo duplican. Donde sí se aprecia una similitud entre ambos sexos es en la frecuencia con la que realizan este consumo, confirmando parcialmente la equiparación en el patrón de consumo obtenida en las últimas investigaciones y encuestas epidemiológicas (Fernández, Dema y Fontanil, 2019; Kuntsche et al., 2011; OEDA, 2018; Simons-Morton et al., 2009).

Es importante resaltar que la elevada frecuencia de realización de esta conducta (más de siete veces al mes) implica un incremento notorio de la probabilidad de aparición de consecuencias biopsicosociales, fijada en algunos estudios a partir de dos o más veces al mes (Anderson, 1996; Livingston, 2013).

La evaluación del patrón de consumo apoya la necesidad de analizar los BD no como un grupo homogéneo, sino como diferentes grupos con niveles de riesgo claramente diferenciados. Este hecho señala una necesaria línea de investigación futura. A parte de concretar al máximo la operacionalización del $\mathrm{BD}$, tal y como se está haciendo en los últimos años, es necesario definir subgrupos dentro de la categoría general, sin olvidar el género.

Un avance importante de cara a disminuir las consecuencias biopsicosociales sería disponer de instrumentos de cribado que permitan detectar el mayor número de jóvenes $\mathrm{BD}$, no solo en contextos académicos, sino también en contextos sanitarios, como Atención Primaria y los gabinetes de salud de los campus universitarios. En estos entornos, sería de gran utilidad disponer de herramientas de detección precoz del BD por la afluencia de jóvenes que visitan estos servicios, y la posibilidad de derivación a recursos especializados en función de la problemática detectada.

Aunque se ha encontrado que el AUDIT-C clasifica a los estudiantes universitarios como BD/NOBD adecuadamente (Cortés et al., 2017b; DeMartini y Carey, 2012; García et al., 2016), el uso del AR2I reduce el número de falsos positivos, ofreciendo una combinación óptima de sensibilidad y especificidad. Este resultado coincide con el obtenido con estudiantes universitarios en el estudio de Cortés et al. (2017a).

En el presente estudio, con un punto de corte de 3 en el AR2I, se detectó aproximadamente el 99\% de los estudiantes universitarios BD. Cuando se utilizó un punto de corte de 4 en hombres y de 3 en mujeres, la capacidad para clasificar BD (sensibilidad) disminuyó únicamente en un punto, pero la capacidad para identificar los NOBD (especificidad) correctamente aumentó notablemente, alcanzando el $100 \%$ en el caso de las mujeres. En todo momento superando además la fiabilidad de la escala original. Dicho resultado no resulta sorprendente, ya que se han utilizado los dos ítems más explicativos del patrón de consumo (Blank et al., 2015), aunque redactados para tener en cuenta las características de BD (Cortés et al., 2017a).

En resumen, los resultados proporcionados por la presente investigación confirman la utilidad de esta nueva 
combinación de ítems para detectar con mayor rapidez y efectividad la población juvenil $\mathrm{BD}$, relevante en recursos asistenciales como la Atención Primaria en los que el tiempo es un factor clave a tener en cuenta. Además, al mostrar su efectividad tanto en población adolescente como universitaria puede concluirse que se trata de un instrumento utilizable en la detección de BD independientemente de la edad y del género. Esto la convierte en una herramienta de screening adecuada para detectar $\mathrm{BD}$ en jóvenes, útil para objetivos clínicos y preventivos y, al mismo tiempo relevante en el campo de la investigación (Arnaud et al., 2016; Walton et al., 2015), ya que una clasificación más ajustada de los sujetos permitirá obtener mayor precisión en los resultados.

Una limitación a tener en cuenta es el uso de autoinformes para determinar el patrón de consumo. No obstante, en la población juvenil, los autoinformes se han considerado válidos y fiables porque aseguran la anonimato y la fiabilidad de los datos, contrariamente a lo que suele suceder con otros tipos de registros, tales como las encuestas enviadas a los hogares (Degenhardt et al., 2013; Knight, Sherritt, Harris, Gates y Chang, 2003).

Otra limitación radica en la generalización de los resultados obtenidos de este estudio, considerando que este consumo está muy presente en la población adulta. Es necesario ampliar la evaluación del AUDIT incluyendo a personas de edades entre 20 y 29 años, el rango con mayor prevalencia de BD en España (OEDA, 2017).

Este trabajo es parte de un estudio más amplio que intenta desarrollar instrumentos de cribado útiles para identificar a jóvenes BD con distintas intensidades de consumo. De manera específica, este trabajo ha mostrado la utilidad del AR2I en diferentes tipos de BD y en grupos de edades concretos.

En futuras investigaciones sería interesante replicar esta adaptación con muestras de otros países ajustando previamente la medida del BD a la UBE del país correspondiente. Esto permitiría disponer de un instrumento de cribado colectivo basado en una definición más rigurosa de $\mathrm{BD}$, facilitando de este modo la comparabilidad de los resultados.

\section{Conflicto de intereses}

Los autores declaran la inexistencia de conflictos de intereses.

\section{Referencias}

Anderson, P. (1996). Alcohol and primary health care. WHO regional publications, European Series, $N^{\circ}$ 64. Copenhagen, Denmark: Word Health Organization Regional Office for Europe.

Arnaud, N., Baldus, C., Elgán, T. H., De Paepe, N., Tønnesen, H., Csémy, L. y Thomasius, R. (2016). Effective- ness of a web-based screening and fully automated brief motivational intervention for adolescent substance use: a randomized controlled trial. Journal of Medical Internet Research, 18, e103. doi:10.2196/jmir.4643.

Babor, T. F., Higgins-Biddle, J. C. y Robaina, K. (2017). USAUDIT, the Alcohol Use Disorders Identification Test, adapted for use in the United States: A guide for primary care practitioners. Rockville, MD: Substance Abuse and Mental Health Services Administration. Recuperado de http:/ / www.ct.gov/dmhas/lib/dmhas/publications/USAUDITGuide_2016.pdf.

Barnett, N. P., Clerkin, E. M., Wood, M., Monti, P. M., Tevyaw, T. O. L., Corriveau, D. y Kahler, C.W. (2014). Description and predictors of positive and negative alcohol-related consequences in the first year of college. Journal of Studies on Alcohol and Drugs, 75, 103-114.

Barry, A. E., Chaney, B. H., Stellefson, M. L. y Dodd, V. (2015). Evaluating the psychometric properties of the AUDIT-C among college students. Journal of Substance Use, 20, 1-5. doi:10.3109/14659891.2013.856479.

Blank, M. L., Connor, J., Gray, A. y Tustin, K. (2015). Screening for hazardous alcohol use among university students using individual questions from the Alcohol Use Disorders Identification Test-Consumption. Drug and Alcohol Review, 34, 540-548. doi:10.1111/dar.12272.

Bowring, A. L., Gouillou, M., Hellard, M. y Dietze, P. (2013). Comparing short versions of the AUDIT in a community-based survey of young people. BMC Public Health, 13, 1. doi:10.1186/1471-2458-13-301.

Brewer, R. D. y Swahn, M. H. (2005). Binge drinking and violence. JAMA, 294, 616-618. doi:10.1001/jama.294.5.616.

Chung, T., Colby, S. M., Barnett, N. P. y Monti, P. M. (2002). Alcohol use disorders identification test: factor structure in an adolescent emergency department sample. $\mathrm{Al}$ coholism: Clinical and Experimental Research, 26, 223-231. doi:10.1111/j.1530-0277.2002.tb02528.x.

Clark, D. B., Gordon, A. J., Ettaro, L. R., Owens, J. M. y Moss, H. B. (2010). Screening and brief intervention for underage drinkers. Mayo Clinic Proceedings, 85, 380-391. doi:10.4065/mcp.2008.0638.

Clark, D. B. y Moss, H.B. (2010). Providing alcohol-related screening and brief interventions to adolescents through health care systems: obstacles and solutions. PLoS Medicine, 7, e1000214. doi:10.1371/journal.pmed.1000214.

Contel Guillamón, M., Gual Solé, A. y Colom Farran, J. (1999). Test para la identificación de trastornos por uso de alcohol (AUDIT): traducción y validación del AUDIT al catalán y castellano. Adicciones, 11, 337-347. doi:10.20882/adicciones.613.

Cortés, M. T., Giménez, J. A., Motos, P. y Sancerni, M. D. (2016). Different versions of the Alcohol Use Disorders Identification Test (AUDIT) as screening instruments for underage binge drinking. Drug and Alcohol Dependence, 158, 52-59. doi:10.1016/j.drugalcdep.2015.10.033. 
Cortés, M. T., Giménez, J. A., Motos, P. y Sancerni, M. D. (2017a). Revision of AUDIT consumption items to improve the screening of youth Binge Drinking. Frontiers in Psychology, 8, 910. doi:10.1016/j.drugalcdep.2015.10.033.

Cortés, M. T., Giménez, J. A., Motos, P., Sancerni, M. D. y Cadaveira, F. (2017b). The utility of the Alcohol Use Disorders Identification Test (AUDIT) for the analysis of binge drinking in university students. Psicothema, 29, 2. doi:10.7334/psicothema2016.271.

Cortés, M. T. y Motos, P. (2015). Cómo definir y medir el consumo intensivo de alcohol. En: M. T. Cortés (Ed.). Guía Clínica. Consumo intensivo de alcohol en jóvenes (pp. 25-46). Barcelona, España: Socidrogalcohol.

Cortés, M. T., Motos, P. y Giménez, J. A. (2015). Consecuencias bio-psico-sociales derivadas del consumo intensivo de alcohol: aspectos psicosociales. En: M. T. Cortés (Ed.). Guía Clínica. Consumo intensivo de alcohol en jóvenes. (pp. 95-120). Barcelona, España: Socidrogalcohol.

Courtney, K. E. y Polich, J. (2009). Binge drinking in young adults: data, definitions, and determinants. Psychological Bulletin, 135, 142-156. doi:10.1037/ a0014414.

de Meneses-Gaya, C., Zuardi, A. W., Loureiro, S. R. y Crippa, J. A. S. (2009). Alcohol Use Disorders Identification Test (AUDIT): An updated systematic review of psychometric properties. Psychology $\mathcal{E}$ Neurosciences, 2, 83. doi:10.3922/j.psns.2009.1.12.

Degenhardt, L., O'Loughlin, C., Swift, W., Romaniuk, J. C., Coffey, C., Hall, W. y Patton, G. (2013). The persistence of adolescent binge drinking into adulthood: findings from a 15-year prospective cohort study. BMJ Open, 3, 1-11. doi:10.1136/bmjopen-2013-003015.

DeMartini, K. S. y Carey, K. B. (2012). Optimizing the use of the AUDIT for alcohol screening in college students. Psychological Assessment, 24, 954-963. doi:10.1037/ a0028519.

Díaz Martínez, L. R., Díaz Martínez, A., Hernández-Ávila, C. A., Fernández Varela, H., Solís Torres, C. y Narro Robles, J. (2009). El consumo riesgoso y dañino de alcohol y sus factores predictivos en adolescentes estudiantes del bachillerato. Salud Mental, 32, 447-458.

ESPAD Group (2016). ESPAD Report 2015: Results from the European School Survey Project on Alcohol and Other Drugs. Luxembourg, Luxembourg: Publications Office of the European Union.

Fernández, M. A., Dema, S. y Fontanil, Y. (2019). The influence of gender roles in alcohol consumption: a qualitative study of adolescents and young adults in Asturias. Adicciones, 31, 260-273. doi:10.20882/adicciones.1003.

García, C. M., Novalbos, R. J., Martínez, D. J. y O'Ferrall, G. C. (2016). Validation of the Alcohol Use Disorders Identification Test in university students: AUDIT and AUDIT-C. Adicciones, 28, 194-204. doi:10.20882/adicciones.775.
Gmel, G., Heeb, J. L. y Rehm, J. (2001). Is frequency of drinking an indicator of problem drinking? A psychometric analysis of a modified version of the alcohol use disorders identification test in Switzerland. Drug and Alcohol Dependence, 64, 151-163. doi:10.1016/S03768716(01)00117-X.

Hagman, B. T. (2016). Performance of the AUDIT in detecting DSM-5 alcohol use disorders in college students. Substance Use and Misuse, 51, 1521-1528. doi:10.1080/10 826084.2016.1188949.

Hanley, J. A. y McNeil, B. J. (1983). A method of comparing the areas under receiver operating characteristic curves derived from the same cases. Radiology, 148, 839-843. doi:10.1148/radiology.148.3.6878708.

Hingson, R., Heeren, T., Zakocs, R., Winter, M. y Wechsler. H. (2003). Age of first intoxication, heavy drinking, driving after drinking and risk of unintentional injury among U.S. college students. Journal of Studies on Alcohol, 64, 23-31.

Hingson, R. W., Zha, W. y Weitzman, E. R. (2009). Magnitude of and trends in alcohol-related mortality and morbidity among US college students ages 18-24, 19982005. Journal of Studies on Alcohol and Drugs, 16, 12-20. doi:10.15288/jsads.2009.s16.12.

Jenkins, M. B., Agrawal, A., Lynskey, M. T., Nelson, E. C., Madden, P. A., Bucholz, K. K. y Heath, A. C. (2011). Correlates of alcohol abuse/dependence in early-onset alcohol-using women. American Journal on Addiction, 20, 429-434. doi:10.1111/j.15210391.2011.00151.x.

Kelly, T. M., Donovan, J. E., Chung, T., Bukstein, O. G. y Cornelius, J. R. (2009). Brief screens for detecting alcohol use disorder among 18-20 year old young adults in emergency departments: Comparing AUDIT-C, CRAFFT, RAPS4-QF, FAST, RUFT-Cut, and DSM-IV 2-Item Scale. Addictive Behaviours, 34, 668-674. doi:10.1016/j. addbeh.2009.03.038.

Knight, J. R., Sherritt, L., Harris, S. K., Gates, E. C. y Chang, G. (2003). Validity of brief alcohol screening tests among adolescents: a comparison of the AUDIT, POSIT, CAGE, and CRAFFT. Alcoholism: Clinical and Experimental Research, 27, 67-73. doi:10.1111/j.1530-0277.2003. tb02723.x.

Kokotailo, P. K., Egan, J., Gangnon, R., Brown, D., Mundt, M. y Fleming, M. (2004). Validity of the Alcohol Use Disorders Identification Test in college students. Alcohol Clinical and Experimental Research, 28, 914-920. doi:10.1097/01.ALC.0000128239.87611.F5.

Kuntsche, E., Kuntsche, S., Knibbe, R., Simons-Morton, B., Farhat, T., Hublet, A., ... Demetrovics, Z. (2011). Cultural and gender convergence in adolescent drunkenness: evidence from 23 European and North American countries. Archives of Pediatrics $\mathcal{E}$ Adolescent Medicine, 165, 152 158. doi:10.1016/j.ijpharm.2011.02.001. 
Livingston, M. (2013). To reduce alcohol related harm we need to look beyond pubs and night clubs. Drug and $\mathrm{Al}$ cohol Review, 32, 113-114. doi:10.1111/dar.12026.

Mallett, K. A., Varvil-Weld, L., Turrisi, R. y Read, A. (2011). An examination of college students' willingness to experience consequences as a unique predictor of alcohol problems. Psychology of Addictive Behaviors, 25, 41. doi: $10.1037 / \mathrm{a} 0021494$.

McCambridge, J. y Thomas, B. A. (2009). Short forms of the AUDIT in a Web-based study of young drinkers. Drug and Alcohol Review, 28, 18-24. doi:10.1111/j.14653362.2008.00010.x.

McKetin, R., Chalmers, J., Sunderland, M. y Bright, D. A. (2014). Recreational drug use and binge drinking: stimulant but not cannabis intoxication is associated with excessive alcohol consumption. Drug and Alcohol Review, 33, 436-45. doi:10.1111/dar.12147.

Mota, N., Álvarez-Gil, R., Corral, M., Holguín, S. R., Parada, M., Crego, A., ... Cadaveira, F. (2010). Risky alcohol use and heavy episodic drinking among Spanish University students: a two-year follow-up. Gaceta Sanitaria, 24, 372-377. doi:10.1016/j.gaceta.2010.02.013.

Ministerio de Sanidad y Consumo [MSC] (2008). Prevención de los problemas derivados del alcohol. En: Conferencia de prevención y promoción de la salud en la práctica clínica en España. Madrid, España: Ministerio de Sanidad y Consumo.

Observatorio Español sobre Drogas y las Adicciones [OEDA] (2017). Informe 2017: EDADES, Encuesta sobre Alcohol y Drogas en España. Delegación del Gobierno para el Plan Nacional sobre Drogas. Madrid, España: Ministerio de Sanidad y Política Social.

Observatorio Español sobre Drogas y las Adicciones [OEDA] (2018). ESTUDES 2016-2017 Encuesta sobre uso de drogas en enseñanzas secundarias en España (avance on-line). Delegación del Gobierno para el Plan Nacional sobre Drogas. Madrid, España: Ministerio de Sanidad y Política Social. Recuperado de http://www.pnsd.msssi.gob.es/ profesionales/sistemasInformacion/sistemaInformacion/pdf/2016_2017_ESTUDES.pdf.

Olthuis, J. V., Zamboanga, B. L., Martens, M. P. y Ham, L. S. (2011). Social influences, alcohol expectancies, and hazardous alcohol use among college athletes. Journal of Clinical Sport Psychology, 5, 24-43. doi:10.1123/jcsp.5.1.24.

Parada, M., Corral, M., Caamaño, F., Mota, N., Crego, A., Rodríguez, S. y Cadaveira, F. (2011). Definición del concepto de consumo intensivo de alcohol adolescente (binge drinking). Adicciones, 23, 53-63. doi:10.20882/ adicciones.167.

Patrick, M. E., Schulenberg, J. E., Martz, M. E., Maggs, J. L., O’Malley, P. M. y Johnston, L. D. (2013). Extreme binge drinking among 12th-grade students in the United States: prevalence and predictors. JAMA Pediatrics, 167, 1019-1025. doi:10.1001/jamapediatrics.2013.2392.
Pilatti, A., Caneto, F., Garimaldi, J. A., Del Valle, B. y Pautassi, R. M. (2013). Contribution of time of drinking onset and family history of alcohol problems in alcohol and drug use behaviors in Argentinean college students. Alcohol and Alcoholism, 49, 128-137, doi:10.1093/alcalc/ agt176.

Read, J. P., Beattie, M., Chamberlain, R. y Merrill, J. E. (2008). Beyond the "binge" threshold: Heavy drinking patterns and their association with alcohol involvement indices in college students. Addictive Behaviors, 33, 225234. doi:10.1016/j.addbeh.2007.09.001.

Rodríguez-Martos, A., Gual Solé, A. y Llopis Llácer, J. J. (1999). The "standard drink unit" as a simplified recording system of alcohol consumption and its measurement in Spain. Medicina Clínica, 112, 446-450.

Seguel, F., Santander, G. y Alexandre, O. (2013). Validez y confiabilidad del test de identificación de los trastornos debidos al consumo de alcohol (AUDIT) en estudiantes de una universidad chilena. Ciencia y Enfermería, 19, 2335. doi:10.4067/S0717-95532013000100003.

Shield, K. D., Gmel, G., Patra, J. y Rehm, J. (2012). Global burden of injuries attributable to alcohol consumption in 2004: a novel way of calculating the burden of injuries attributable to alcohol consumption. Population Health Metrics, 10, 9. doi:10.1186/1478-7954-10-9.

Simons-Morton, B. G., Farhat, T., Ter-Bogt, T. F., Hublet, A., Kuntsche, E., Gabhainn, S. N., ... Kokkevi, A. (2009). Gender specific trends in alcohol use: cross-cultural comparisons from 1998 to 2006 in 24 countries and regions. International Journal of Public Health, 54, 199-208. doi:10.1007/s00038-009-5411-y.

Thomas, B. A. y McCambridge, J. (2008). Comparative psychometric study of a range of hazardous drinking measures administered online in a youth population. Drug and Alcohol Dependence, 96, 121-127. doi: 10.1016/j.drugalcdep.2008.02.010.

Unión Europea (2010). Special Eurobarometer 331: EU citizens' attitudes towards alcohol. Brussels, Belgium: European Union.

Vargas-Martínez, A. M., Trapero-Bertran, M., Gil-García, E. y Lima-Serrano, M. (2018). Impact of the Binge Drinking (BD) in Adolescence. Are we doing it right? Adicciones, 30, 152-154. doi:10.20882/adicciones.1033.

Walton, M. A., Chermack, S. T., Blow, F. C., Ehrlich, P. F., Barry, K. L., Booth, B. M. y Cunningham, R. M. (2015). Components of brief alcohol interventions for youth in the emergency department. Substance Abuse, 36, 339-349. doi:10.1080/08897077.2014. 